\title{
Microlearning: innovaciones instruccionales en el escenario de la educación virtual
}

\author{
Microlearning: instructional innovations in virtual education scenario
}

\author{
Fabiola Salas Díaz \\ Edgar Oswaldo González Bello \\ Etty Haydeé Estévez Nénninger
}

\begin{abstract}
RESUMEN
El microlearning hace referencia a un aprendizaje breve, conciso, frecuentemente digital y móvil; por su versatilidad en medios y formatos, así como por las posibilidades de aplicación bajo distintas pedagogías, representa una alternativa de innovación instruccional conectada con las prácticas de la era digital, demandada por la educación virtual en escenarios de la educación superior. El propósito del estudio fue comprender el desarrollo de innovaciones relacionadas con enfoques teóricos utilizados como sustento analítico del microlearning para entornos virtuales; se hizo diferencia de las etapas del proceso innovador a partir de elementos del conocimiento reportados por la producción científica. Se utilizó un enfoque metodológico teórico documental dirigido a recopilar, analizar y categorizar las investigaciones durante las distintas etapas de la innovación. Los hallazgos manifestaron un escaso uso de teoría para sustentar las investigaciones, aunque también destacaron los estudios situados en una etapa de gestación de la innovación, en la que los aportes al conocimiento empírico fueron sobre análisis teóricos y modelos de implementación. Se concluyó que el análisis y la perspectiva evolutiva de los avances en microlearning ofrecen las bases para sustentar teóricamente las innovaciones instruccionales, necesarias desde la perspectiva de los estudiantes, por la creciente interacción tecnológica a la que están expuestos.
\end{abstract}

Palabras clave: innovación educacional, enseñanza superior, aprendizaje en línea.

\begin{abstract}
Microlearning refers to short, concise, often digital and mobile learning; due to its versatility in media and formats, as well as the possibilities of its application under different pedagogies, it represents an alternative of instructional innovation connected with Digital Era practices, demanded by virtual education in higher education scenarios. Therefore, the aim of this work was to understand the development of innovations in relation to the theoretical approaches used as analytical support of microlearning for virtual environments, differentiating the stages of the innovative process based on elements of reported scientific production knowledge. A documentary theoretical methodological approach was used aimed at compiling, analyzing and categorizing research under different innovation stages. Findings showed little use of theory to support research, although studies referring to an initial stage of innovation also stood out. At this stage contributions to empirical knowledge were on theoretical analyses and implementation models. It is concluded that the analysis and evolutionary perspective of microlearning advances present the bases to theoretically support instructional innovations, required from the students' perspective, due to the growing technological interaction to which they are exposed.
\end{abstract}

Keywords: educational innovation, higher education, online learning. 


\section{INTRODUCCIÓN}

En el ámbito de la educación superior, más allá de integrar medios digitales en las aulas como respuesta a los cambios tecnológicos, económicos y sociales, se requieren conceptos que sustenten y ofrezcan orientación a la enseñanza, pero también estrategias específicas para que la educación virtual cumpla con su definición, y especialmente con la posibilidad de desarrollar competencias para afrontar retos profesionales en el estudiante. Por este motivo, la UNESCO (2020) refiere que el aprendizaje continuo es una competencia clave que habría de abordarse a través de la transformación de los programas en las instituciones de educación superior (IES), al ampliar las posibilidades de la educación mediante la introducción de modalidades blended y particularmente el uso de microlearning.

El microlearning es un término que recoge diversas posturas: algunas se relacionan con mejorar los aprendizajes utilizando medios digitales, otras un tanto más ideológicas sobre aprender en un mundo más acelerado producto de cambios tecnológicos, y algunas más vinculadas con la educación o la formación, donde se conceptualizan procesos de aprendizaje a partir de perspectivas micro (Hug, 2012). A pesar de estas diferencias, de forma generalizada se hace alusión a un aprendizaje flexible a través de contenidos breves y concisos, accesible en cualquier momento y lugar, por lo que comúnmente suele vincularse con tecnologías digitales y móviles.

Para la docencia universitaria, el microlearning es considerado como práctica innovadora que forma parte del grupo de tecnologías para el aprendizaje (Becker, Brown, Dahlstrom, Davis, DePaul, Diaz y Pomerantz, 2018), la cual suele abordarse a través del diseño instruccional. En este sentido, el informe Horizon sobre la educación superior, entre las prácticas y tecnologías emergentes expuestas, presenta la elevación del diseño instruccional que, según se reporta, es la de mayor impacto en el aprendizaje (Educause, 2020). Entonces, es notable que el microlearning ofrece un espectro de posibilidades para desarrollar materiales didácticos al permitir variaciones

Fabiola Salas Díaz. Universidad de Sonora, México. Es maestra en Ciencias de la Computación por el Tecnológico Nacional de México e ingeniera en Sistemas de Información por el Instituto Tecnológico y de Estudios Superiores de Monterrey. Actualmente estudia en el programa de doctorado en Innovación Educativa en la línea de investigación de Estudiantes y Tecnología en Educación Superior. Correo electrónico: fasadi@hotmail.com. ID: https://orcid.org/0000-0001-5202-0247.

Edgar Oswaldo González Bello. Profesor-investigador titular de tiempo completo en el Departamento de Psicología y Ciencias de la Comunicación en la Universidad de Sonora, México. Es doctor en Ciencias Sociales y maestro en Innovación Educativa. Es miembro del Consejo Mexicano de Investigación Educativa y cuenta con los reconocimientos al perfil ProDEP y del Sistema Nacional de Investigadores. Correo electrónico: edgar.gonzalez@unison.mx. ID: https://orcid.org/0000-0001-6297-2516.

Etty Haydeé Estévez Nénninger. Profesora-investigadora de tiempo completo en el Departamento de Psicología y Ciencias de la Comunicación en la Universidad de Sonora, México. Es doctora en Ciencias con Especialidad en Investigaciones Educativas por el IPN-CiNVESTAV y maestra en Educación por el Instituto Tecnológico y de Estudios Superiores de Monterrey. Es miembro del Consejo Mexicano de Investigación Educativa. Tiene los reconocimientos al perfil ProdeP y del Sistema Nacional de Investigadores. Correo electrónico: ettyestevez@gmail.com. ID: https://orcid.org/0000-0002-0105-2369. 
en formato, medio o tiempo, y otras características más; esto hace posible valorarlo como una innovación instruccional de los ambientes virtuales que se desarrollan para la educación superior.

Las innovaciones instruccionales, como toda innovación, buscan marcar una diferencia y parten de un propósito. En el ámbito de la educación, las intenciones para innovar son claras, están dirigidas a qué se quiere lograr con los estudiantes; por lo tanto, el desarrollo de innovaciones se propone, según Rivas, “alterar los elementos de un orden escolar que apagan o limitan el deseo de aprender de los alumnos" (Rivas, 2017, p. 19). Tomando en cuenta el propósito de las innovaciones, las que se relacionan con la instrucción en los entornos virtuales suelen dejarse de lado, sobre todo al considerar que, de los diversos factores que convergen al utilizar estos entornos, la enseñanza es concebida desde la educación en formato presencial. Esta situación es común en universidades que tradicionalmente han ofrecido educación en modalidad presencial y buscan extender su oferta a través de estrategias virtuales.

De igual manera se visibiliza en la situación experimentada en las IES debido al brote de la enfermedad por coronavirus (COVID-19), cuando se inició abruptamente una migración de las actividades presenciales a los entornos virtuales. A pesar de la existencia -pero también del desarrollo- de tecnología que posibilitara la continuidad de la educación superior, el cómo enseñar en el medio virtual quedó en cierta medida desatendido, pues la escasez de alternativas instruccionales de dominio del docente en la virtualidad suele favorecer estrategias de mayor pertinencia para la educación presencial. La desafección de los estudiantes por el cambio de modalidad podría ser consecuencia de esto, dado que la UNESCO-IESALC (2020) refiere que esto se debe principalmente a los contenidos de los cursos, que, más que adoptar diseños propios para entornos virtuales, intentan dar solución a la problemática suscitada.

Por tanto, se percibe la oportunidad de revalorar las innovaciones instruccionales en los espacios virtuales, pues aún fuera del contexto de la pandemia, para muchas instituciones de educación superior, digitalizar contenidos se volvió praxis habitual al introducir entornos virtuales como innovación educativa, de tal forma que inicialmente se sentaron las bases para desplegar un proceso que requería ser ajustado en aras de asegurar su viabilidad. Esto no sucedió en todos los casos, por lo que las características de estos ambientes en ocasiones aluden a la innovación burocratizada (Aguerrondo, 1992), con todos los elementos de permanencia, pero con matices limitados de la innovación que se requiere en la enseñanza. Los entornos virtuales existen en el discurso como medios innovadores por sí mismos, sin embargo, a veces distan de serlo; por este motivo representan un espacio de interés para la innovación instruccional.

Para Lee (2008), la innovación instruccional tiene el propósito de entender las diferencias particulares de los estudiantes, de forma tal que, mediante el desarrollo o la adopción de métodos de enseñanza diversificados, crezca el interés en el aprendizaje 
y, por tanto, mejoren los efectos producidos por el estudio. El microlearning como área de investigación aborda estas cuestiones, por lo que en este ámbito, un enfoque de la innovación con énfasis en el proceso, refleja los componentes de las innovaciones desde una perspectiva en cierto modo gradual.

A pesar de las intenciones anteriores, ha de entenderse que las innovaciones no se refieren a una secuencia de etapas forzosamente transitables, sino al ajuste y la transformación de algún elemento del sistema educativo (Aguerrondo, 1992). Bajo esta premisa, los ajustes asociados al microlearning, desde la perspectiva de Elmore (1990, citado en Zabalza, 2004), aluden a la reforma de la enseñanza con base en elementos tecnológicos, en la cual se involucran elementos técnicos, pero también de instrucción, metodología, planeación o evaluación.

La perspectiva teórica de la innovación es, por tanto, un marco de análisis apropiado para el microlearning, puesto que ofrece distintas miradas desde las cuales es posible la construcción de bases para la viabilidad de su despliegue en entornos virtuales de la educación superior. La propuesta por Aguerrondo (1992) se considera entonces como un referente pertinente, pues dirige el análisis de las innovaciones hacia los momentos en que se sitúa el proceso de innovación, a partir de una clasificación de tres etapas: gestación, ejecución y desarrollo; esta perspectiva contempla a procesos internos más característicos que se dan al innovar, dando lugar a una lógica de representación de sus esfuerzos, más que a una secuencia real de cómo llevarlos a cabo.

La etapa de gestación comprende tres posibles momentos: el primero da cuenta de una disfunción en algún aspecto de la educación, y también de las ideas, modelos o soluciones posibles; un segundo momento identifica la coyuntura para la innovación y los actores clave que influirían en el proceso; concluye con un tercer momento que la autora refiere a la elaboración de la propuesta.

En cuanto a la etapa de ejecución, esta se desarticula en tres viabilidades: la primera político-cultural, que se asocia a los grupos o comunidades donde incide la innovación; la segunda organizativo-administrativa, que comprende aquellos aspectos burocráticos que intervienen, y por último la viabilidad material, en la cual se valoran recursos humanos, tiempo y recursos materiales. Esta etapa también responde a los aspectos de incidencia en la innovación que facilitarán su permanencia o, en cierta medida, el reconocimiento y forma de afrontar para evitar la ruptura del proceso. En un sentido más estricto, esta etapa se describe más por la influencia de grupos de poder y su injerencia en frenar o propiciar la innovación.

Por último, la etapa de desarrollo contempla tres vías posibles que podría tomar la innovación: consolidación, burocratización e interrupción. Los desarrollos en etapa de consolidación son procesos que permanecen y que suelen verse enriquecidos. Las innovaciones que siguen la vía de la burocratización son experiencias que permanecen en la formalidad, pero han perdido el aspecto innovador. La interrupción, por último, comprende las experiencias que llegan a su fin por disposiciones formales. 
Al estimar la diversidad de esfuerzos y uso de referentes teóricos que buscan el desarrollo de innovaciones desde las características que describen al microlearning, resulta de interés la estructura de análisis de las etapas propuestas por Aguerrondo (1992), puesto que permite observar matices de diferenciación con relación al mismo proceso de innovación, en función de los estudios e investigaciones que se han desarrollado. Asimismo, la estrategia de ordenar en las etapas de innovación descritas permite examinar este campo de estudio como un todo y ofrece una valoración de su proceso evolutivo en un espacio de tiempo, por medio de fases diferenciadas.

Desde una perspectiva analítica, este estudio tiene como objetivo comprender el desarrollo de innovaciones relacionadas con los enfoques teóricos utilizados como sustento analítico del microlearning para entornos virtuales, distinguiendo las etapas del proceso innovador con base en elementos del conocimiento reportado por la producción científica.

\section{Metodología}

El estudio sigue una metodología documental, caracterizada por ser un proceso de análisis de información a partir de diversas fuentes. Este análisis se realiza en varias etapas del proceso: recolectar, seleccionar y analizar la información para finalmente darla a conocer de manera ordenada (Pimienta y De la Orden, 2017). A través de fases, el análisis descompone el objeto de estudio en partes y al final se ofrece una síntesis ordenada de la información y su resultado.

Como fase inicial de la revisión documental se identificaron diversas investigaciones sobre microlearning, considerando únicamente fuentes primarias de carácter científico. Al ser escasos los estudios presentados en peer-reviewed journals, se optó por ampliar los resultados omitiendo restricciones sobre el tipo de revista. Como criterio de delimitación se seleccionaron publicaciones efectuadas del año 2016 en adelante. Se consultaron bases de datos nacionales e internacionales, discriminando de los hallazgos las investigaciones meramente técnicas como son frameworks, desarrollo y optimización de aplicaciones, arquitecturas, sistemas inteligentes, entre otras. De este recorte se exceptuaron las investigaciones técnicas que contemplan como parte de los objetivos valoraciones del usuario, mediciones de desempeño o actitudes hacia el uso de desarrollos específicos. Los resultados arrojan una cantidad considerable de investigaciones en el continente asiático, sin embargo, para efectos de este análisis y como otro de los criterios, solo se tomaron en cuenta estudios reportados en inglés y en español.

Una vez seleccionados los trabajos, se determinaron características de diferenciación derivadas de la clasificación de la innovación que propone Aguerrondo (1992). Las tres etapas de un proceso de innovación se dividen a su vez en tres posibles momentos, sin embargo, por las características del medio por el cual se reporta cada 
innovación, algunas subclasificaciones son difícilmente observables, o bien están presentes en todos los casos. Por este motivo se omitieron algunas subcategorías, quedando las etapas primera y segunda, con dos subclasificaciones, mientras que para la etapa de desarrollo solo se consideró observable la subcategoría de consolidación de la innovación. Una vez establecidas estas consideraciones, los artículos se analizaron y clasificaron conforme a las categorías resultantes.

Los elementos observables para ubicar las investigaciones en cada categoría son los siguientes: el tipo de estudio, el objetivo, la población de análisis y la cantidad de tiempo en que se ha llevado a cabo. Además de estos elementos, otras cuestiones particulares de cada subclasificación se tomaron en cuenta. En cuanto a las investigaciones en la subclasificación de ideas, modelos o soluciones, por lo general son documentales, explican los beneficios del microlearning y pueden contemplar un modelo, sin embargo, con respecto a los modelos, no se informa con precisión la interrelación de sus partes. En el caso de la subcategoría de propuestas, los estudios comprenden guías claras de despliegue o modelos que profundizan en sus elementos e interrelaciones.

La coyuntura para la innovación que se sitúa en la primera fase se considera en este caso como elemento fundamental de todas las investigaciones, por lo que esta característica está implícita en todas las etapas. Se identifica en los artículos a partir del propósito del estudio y se reporta en cada etapa de la innovación. Respecto a la viabilidad político-cultural, referida en la segunda etapa del proceso de innovación, se identifica como parte de las intenciones del estudio al reportar que el elemento humano funge como factor de influencia en el uso de objetos de microlearning; principalmente se asocia a los docentes en este ámbito.

La viabilidad material contempla estudios de implementaciones cuyo propósito estuvo dirigido a establecer este tipo de viabilidad a través del medio, los materiales didácticos y los actores del proceso educativo. Se exceptúan de esta categoría los trabajos que presentan particularidades de la viabilidad político-cultural. Por último, la subclasificación de consolidación en la etapa de desarrollo comprende los trabajos que superan un ciclo educativo ejecutándose. Las dos últimas subclasificaciones se omiten puesto que el tipo de investigaciones analizadas no suelen reportar estas cuestiones. La tabla 1 muestra las características analizadas para cada subclasificación de la innovación.

De esta forma, un total de 58 artículos fueron analizados y distribuidos entre las subclasificaciones de innovación: ideas, modelos o soluciones $28 \%$, propuestas $24 \%$, viabilidad político-cultural 3\%, viabilidad material 34\% y consolidación 11\%. La etapa de gestación recoge el 52\% de los resultados, mientras que las etapas de ejecución y desarrollo representan el 37\% y el 11\% respectivamente. Las particularidades de cada grupo se describen a continuación. 
Tabla 1. Elementos de análisis por etapa y subclasificación de la innovación.

\begin{tabular}{lll}
$\begin{array}{c}\text { Etapa de la } \\
\text { innovación }\end{array}$ & \multicolumn{1}{c}{ Subclasificación } & \multicolumn{1}{c}{ Elemento de análisis } \\
\hline Gestación & Ideas, modelos o soluciones & $\begin{array}{l}\text { Investigaciones documentales que exponen los beneficios del microlearning } \\
\text { Modelos que no precisan la interrelación entre sus componentes }\end{array}$ \\
\hline Ejecución & Elaboración de la propuesta & Guías claras de despliegue y modelos que explican sus elementos e interrelaciones \\
\cline { 2 - 3 } & Viabilidad político-cultural & Influencia de las personas para hacer uso de objetos de microlearning \\
\cline { 2 - 3 } & Viabilidad material & $\begin{array}{l}\text { Innovaciones que valoran medios distintos, usos alternos a medios } \\
\text { existentes, propuestas o adaptación a material didáctico e influencias } \\
\text { de los actores educativos }\end{array}$ \\
\hline Desarrollo & Consolidación & Resultados de despliegues con al menos un ciclo educativo en ejecución \\
\hline
\end{tabular}

Fuente: Construcción propia con base en la clasificación propuesta por Aguerrondo (1992).

\section{Resultados}

\section{Gestación: del origen de la innovación a la planificación de las propuestas}

En esta categoría se concentran estudios teóricos o propuestas de modelos; los aportes están dirigidos mayormente a la caracterización de los elementos necesarios para el desarrollo de contenidos con microlearning. En la primera subclasificación, referente a ideas, modelos o soluciones, las investigaciones en general se posicionan en un espacio amplio para la innovación, en el cual se carece de precisar las necesidades específicas de una población u objeto y las propuestas innovadoras parten de generalizar nuevas formas de aprender, pero con un escaso sustento teórico. Suele justificarse la innovación a través de las características propias del microlearning, en algunos casos relacionándolas con otros enfoques, como micro-credenciales, el aprendizaje a lo largo de la vida (lifelong learning) o los cursos en línea abiertos y masivos (Massive Open Online Course, MOOC).

Destacan por el uso de teorías estudios como el de Busse y Schumann (2019), quienes, con el propósito de descubrir los requerimientos didácticos del microlearning, presentan la metodología para un estudio empírico. En su propuesta consideran teorías del aprendizaje, teorías de aprendizaje multimedia y teorías de diseño instruccional. En particular se plantea el estudio de los siguientes enfoques: cognitivismo, constructivismo, conductismo, teoría de la carga cognitiva, teoría del aprendizaje multimedia, el modelo integrado de comprensión de análisis y textos, teoría de presentación de componentes, teoría de la elaboración, 4C/ID, y la instrucción anclada. El alcance del estudio implica una fase de análisis de estas teorías, considerando además entrevistar a diseñadores instruccionales. La fase final de la propuesta metodológica es la integración de los resultados obtenidos para derivar una propuesta de elementos didácticos para el microlearning. 
Otro elemento teórico identificado dentro de modelos, ideas y soluciones lo propone Shail (2019); él realiza un análisis de los beneficios del microlearning en el aprendizaje. A través de la curva del olvido de Ebbinghaus sustenta teóricamente la retención de contenidos de aprendizaje. Esta teoría sostiene que la capacidad de retención se reduce con el tiempo, pudiendo llegar a perderse $50 \%$ de lo aprendido después de 20 minutos de terminar el proceso, y seguir perdiendo información conforme pasa el tiempo. El enfoque del autor se dirige hacia aspectos cognitivos y de retención, bajo el cual argumenta que cada cerebro trabaja a su propio ritmo los procesos de aprendizaje. Se propone utilizar microlearning para incidir en la memoria a corto y largo plazo, sugiriendo resultados favorables hacia esta metodología en particular, sobre todo al asociarla con dispositivos móviles como elemento clave en el aprendizaje de los jóvenes, ya que esta combinación ofrece flexibilidad en tiempo, espacio y ritmo de trabajo.

En un último momento, la etapa de gestación comprende las propuestas de innovación. Para esta subcategoría de análisis se recogen los estudios que presentan un análisis teórico con derivación de lineamientos específicos para ejecutar la innovación. Aunque en este momento de la gestación las investigaciones son más de carácter teórico y carecen de mediciones o experimentación, se percibe menor amplitud en las propuestas. El espacio para la innovación es un tanto más preciso y en una alta proporción se enfoca a directrices para el diseño de cursos, o bien a recomendaciones para la transformación de cursos de e-learning a microlearning con procesos o fases claramente delimitadas.

Respecto a las teorías utilizadas, sobresale el trabajo de Göschlberger (2016), cuyo soporte teórico es la espiral de competencias de Baumgartner compuesta por tres tipos de aprendizaje: el aprendizaje I sucede al adquirir conocimientos básicos de un tópico o materia; el aprendizaje II se da cuando el aprendizaje se puede adquirir de manera autodeterminada; finalmente el aprendizaje III se presenta con la construcción de conocimiento por parte del estudiante. El autor se propone aplicar la teoría en el diseño de una plataforma de microlearning social, con cuatro elementos estructurales: (1) crear y compartir; (2) evaluar, comentar y mejorar; (3) etiquetar y recolectar, y (4) interactuar y resolver.

De igual manera, Meng, Wang y Li (2016) proponen diseñar cursos para microlearning con base en la teoría de la carga cognitiva, la cual hace referencia a dos tipos de memoria: la memoria a largo plazo, que no tiene restricción, y la memoria a corto plazo, la cual es limitada y almacena información durante el proceso de aprendizaje. Concretan los aspectos de diseño con base en las cargas cognitivas asociadas a la memoria a corto plazo: intrínseca, extrínseca y relevante. Además contemplan niveles de aprendizaje en asignaturas, con el fin de que el estudiante interactúe con los materiales de aprendizaje según se decida. Puesto que cada estudiante acepta distintos niveles de 
carga cognitiva, esta teoría es efectiva para desarrollar cursos para dispositivos móviles.

Otra perspectiva teórica que presentan Meng y Li (2016) es la aplicación del modelo de Koole para estudiar la factibilidad del microlearning en estudiantes. El modelo está formado por tres círculos que intersecan en el aprendizaje móvil: usabilidad, estudiante y aspectos sociales. De forma excepcional en este grupo de innovaciones, se incluye este trabajo que reporta mediciones. Mediante un cuestionario se determinan las posibilidades de uso de microlearning, considerando el modelo de Koole, sin embargo, los elementos propios de microlearning se contemplan de forma superficial y prevalece un enfoque acentuado hacia el aprendizaje móvil más que sobre las particularidades del microlearning.

En suma, este tipo de acciones innovadoras que convergen en un proceso de gestación se caracterizan por ser modelos teóricos para sustentar la incorporación de microlearning a los entornos de aprendizaje virtual. Exponen la necesidad de innovación al sugerir formas más flexibles de aprender y tal vez más adaptables a los contextos de los estudiantes. Sobre todo se destaca su pertinencia al asociar el microlearning con enfoques de aprendizaje novedosos que son vinculados con la tecnología, dirigidos a fomentar en los estudiantes habilidades de autonomía para un aprendizaje continuo.

\section{Ejecución: propuestas y construcción de viabilidades para la innovación}

Dentro de la subcategoría de viabilidad político-cultural se concentran estudios empíricos que claramente presentan la intención de convencer a través de la práctica, incidiendo en la viabilidad para el éxito y la generalización de la innovación. La coyuntura para la innovación en este grupo de viabilidad se hace patente cuando en los objetivos de investigación se establece la motivación de los docentes para llevar el microlearning a la práctica. Los estudios en esta etapa se dirigen al desarrollo profesional docente, formándolos a través de microlearning, en aras de trasladar su incursión a las aulas.

Solo se identifica un trabajo con sustento teórico en esta subcategoría, en el que Shamir-Inbal y Blau (2020) presentan los resultados de un estudio para medir la motivación a través de un curso con microlearning para el desarrollo profesional docente. Con base en el modelo de aprendizaje autorregulado (RSRLM, por sus siglas en inglés) se examinaron estrategias de autorregulación, y los retos que el microlearning representa para los líderes de tecnologías. Se reporta un mayor sentido de competencia de los participantes, también un aumento en la motivación al aprendizaje por el formato del curso. Los resultados sugieren que este tipo de pedagogía innovadora es apropiada para el contexto analizado, mejora la percepción sobre el aprendizaje y se espera que, al haberla probado, los profesores transmitan la experiencia con sus estudiantes.

El segundo momento de la etapa de ejecución o viabilidad material, identifica en las investigaciones desarrollos novedosos o implementaciones de cursos, a partir de los cuales se analizan cuestiones como aprendizaje, usabilidad, motivación, o 
elementos de repercusión en las posibilidades de consolidación de las innovaciones instruccionales con microlearning. El espacio para la innovación se vuelve de mayor precisión, las implementaciones están enfocadas, en gran medida, hacia el desarrollo de aplicaciones móviles para el aprendizaje de idiomas y tecnología. En esta subcategoría se registran estudios que se complementan con enfoques novedosos de aprendizaje, aunque algunos también carecen de soporte teórico que ofrezca explicación y sustento al microlearning.

Con respecto al sustento teórico de las investigaciones, se sitúan estudios como el de Nikou y Economides (2018), que mediante el desarrollo de un ambiente de aprendizaje implementan el microlearning en una asignatura de ciencias para desarrollar tareas y actividades después de cada clase. Con base en la teoría de la autodeterminación de la motivación, buscan incrementar la motivación de los estudiantes. La teoría distingue entre la motivación intrínseca y la extrínseca, la primera se asocia al bienestar y al mejor rendimiento, y para mejorarla se necesita de autonomía, competencia y relaciones. El estudio integra estos elementos para medir la motivación y reportan en los estudiantes un mayor sentido de autonomía, mejora en la percepción de sus competencias al desarrollar las tareas y la facilidad de interacción entre los miembros de clase.

También, valorando ciertos elementos motivacionales, Göschlberger y Bruck (2017) desarrollan una aplicación para promover el aprendizaje con base en los principios de la "gamificación" (gamification) y el microlearning. Ellos utilizan como marco de referencia el octágono (octalysis) de la motivación, asociado con frecuencia a la gamificación. Las ocho dimensiones que forman los lados del octágono y estudian la motivación en los entornos lúdicos son los siguientes: significado, creatividad, influencia social, imprevisibilidad, pérdida, escasez, propiedad y realización. Se mide la motivación extrínseca a partir de los datos generados por el uso de la aplicación y se concluye un incremento en la motivación mediante la combinación de microlearning y los elementos lúdicos a través del aprendizaje móvil.

Almazova, Rogovaya y Gavrilova (2018), por otra parte, presentan un estudio del uso de microlearning como soporte al aprendizaje de idiomas. Sustentan la implementación en la teoría de generaciones de William Strauss y Neil Hove, al establecer un cambio generacional aproximadamente cada 22 años. Ellos utilizan el microlearning con base en esta teoría y en el "pensamiento de mosaico", que describe jóvenes hábiles para trabajar cantidades grandes de información, la preferencia por información visual, con un decremento en la atención y en la capacidad de análisis, entre otros aspectos. Utilizan microlearning a través de pósters e infográficos como incentivo de comunicación entre los estudiantes.

Desde la teoría del constructivismo, Zhou y Deng (2018) evalúan la enseñanza del aula invertida en combinación con microlearning. El constructivismo sostiene que 
el aprendizaje se da de forma activa cuando el estudiante construye el conocimiento y no lo acepta pasivamente. Los autores aplican el modelo desarrollado en una asignatura de tecnología y concluyen mejores resultados con este modelo de enseñanza.

En conclusión, los estudios analizados se sitúan en esta etapa de la innovación puesto que valoran las posibilidades de implementación, bien sea por el sustento en el uso del recurso tecnológico o por la preferencia de los estudiantes hacia el uso de determinadas implementaciones. Sin embargo, cabe destacar que no se reporta si las viabilidades correspondientes han sido consideradas para su permanencia. Se asocian a esta clasificación desde el criterio de ejecución por un tiempo determinado, y sobresale la característica de presentarlas sin referencia a una teoría en específico. El sustento teórico en aproximadamente $80 \%$ de los casos se refiere a la definición o características del microlearning.

En algunos casos se implementan acciones con base en las características propias del microlearning y en su relación estrecha con otras tendencias educativas novedosas como son los MOOCs, la gamificación, o las redes sociales. Son representativos de esta situación casos como el de Coakley, Garvey y O’Neill (2017), cuya implementación se sustenta en el desarrollo profesional continuo y el aprendizaje a lo largo de la vida; asimismo la propuesta de King (2018), que utiliza microlearning y analíticas de Twitter para promover el compromiso con los programas innovadores de ingeniería.

\section{Desarrollo: consolidación de la innovación}

El desarrollo de innovaciones comprende investigaciones que se han afianzado y que han conseguido prosperar, incluso hacer reajustes para su permanencia. La coyuntura para la innovación en estos proyectos es aún más precisa, aunque también el impacto es de mayor alcance. En general, son experiencias de programas gubernamentales que han tenido resultados favorables. Al igual que sucede en las etapas previas, el microlearning tampoco se analiza necesariamente como foco central, sino que suele integrarse con otros enfoques de aprendizaje.

Por los elementos teóricos que presentan, son relevantes el trabajo de Friedler (2018) y la aportación de Allela, Ogange, Junaid y Prince (2019). Ambos se consideran en consolidación, pues refieren continuidad en la innovación. Por una parte, Friedler (2018) presenta resultados de un programa piloto nacional para formación docente que ofrece cursos con la metodología del microlearning y micro-credenciales, bajo el sustento de la teoría del aprendizaje basado en competencias. La estructura de los cursos dota al docente de autonomía en el aprendizaje, y las micro-credenciales respaldan las competencias adquiridas. Reporta niveles altos de aprovechamiento y aceptación por los docentes hacia este tipo de formación.

Por otra parte, Allela et al. (2019) evalúan el efecto de un programa de formación profesional docente en el cual se utilizó microlearning y dispositivos móviles para 
mejorar la educación virtual. Los elementos teóricos comprenden la caracterización del microlearning y las comunidades de práctica (CoP por sus siglas en inglés). Se complementa con elementos del aprendizaje a lo largo de la vida (lifelong learning), aprendizaje fragmentado y autoaprendizaje, que atienden en conjunto las necesidades específicas de los profesores en formación a través de la educación virtual. El uso de dispositivos móviles es un elemento clave para este despliegue. Los resultados indican mayor motivación hacia el aprendizaje, también la necesidad de seguir evaluando el programa en curso.

Las innovaciones instruccionales, que se caracterizan por su continuidad, son proyectos que han encontrado viabilidad sostenida en el uso de microlearning; la mitad de ellos pertenecen a programas gubernamentales. Entre los estudios que no son gubernamentales se encuentra el de Skalka y Drlilk (2020), donde no fue posible recuperar una teoría asociada al microlearning pero destaca la continuidad de su uso en asignaturas de educación superior. Los autores presentan resultados sobre un estudio predictivo de estudiantes en riesgo de abandono o reprobación. Se analizan cuatro ciclos escolares de la asignatura de programación. Los resultados respecto al microlearning reportan que los estudiantes mencionan con mayor frecuencia una mejor comprensión del contenido, información concisa y rápida, así como mayores posibilidades de práctica. Este caso es característico de lo que Aguerrondo (1992) considera como una innovación consolidada, pues refiere no solo la continuidad sino el enriquecimiento de las actividades de microlearning año con año, refinando los elementos de innovación para su permanencia.

Las distintas etapas en que se situaron los esfuerzos hacia la innovación instruccional con microlearning comprenden estudios que por sus particularidades se asocian mayormente a la dimensión tecnológica de la innovación (Tejada, 1998). Estos estudios coinciden en la búsqueda de la transformación de la enseñanza en entornos virtuales a través de diseños o implementaciones sustentadas en gran medida por las funcionalidades propias de una tecnología. También es visible cómo ha ido creciendo el interés por estudios que buscan refinar los elementos didácticos de esta metodología, al igual que la preocupación por el establecimiento de modelos o procedimientos para migrar de un escenario de e-learning a uno de microlearning.

\section{Discusión}

Los resultados de las investigaciones obedecen en parte a acciones planeadas por iniciativas particulares o propuestas de modelos didácticos vinculados mayormente, más no exclusivamente, a innovaciones micro. Se observa también que una porción considerable de las investigaciones sobre microlearning carece de una base teórica, o más bien, el sustento es dirigido a describir las características de la metodología. Destacan, sobre todo en la fase de ejecución, las experiencias aisladas de implemen- 
tación. Sobresale el aspecto técnico de algunos estudios, especialmente en la fase de implementación, que hacen énfasis en las características del medio y reportan escasos elementos instruccionales. Se dejan ver en menor medida estudios que reducen el énfasis en el medio, mas no deja de ser un factor crucial que se reporta, y que analizan otros elementos de incidencia en la instrucción de la educación virtual con esta metodología. Además, el alcance del conjunto de innovaciones que se reporta permite advertir si su origen obedece más a exigencias que reciben las instituciones y las presiones a los sistemas educativos (Zabalza, 2004), puesto que en su mayoría distan de concretarse.

De forma general y como coyunturas para la innovación se identifican la influencia de la tecnología, especialmente la móvil, la necesidad de aprendizajes flexibles, el desarrollo de autonomía en los estudiantes y el uso combinado con otras tendencias novedosas para el aprendizaje. Entonces, esto orienta a cuestionar si estas se producen solo formalmente y sin la capacidad de impacto sobre las prácticas reales (Zabalza, 2004), aunque también inferir que empiezan a construirse bases para la incorporación de tecnología en los entornos virtuales, contemplando elementos del aprendizaje profundo (Fullan, 2013). Es más evidente en la etapa de desarrollo o consolidación de la innovación, en la cual la intención de conectar a los estudiantes con el propósito del aprendizaje y el desarrollo de autonomía a través del microlearning es más clara.

Por otra parte, se hace notar que, en función de la etapa a la que se asocia el proceso innovador, el grado de especificidad de los objetivos varía; así, en etapas tempranas de la innovación los objetivos abordan el tema con amplitud, mientras que en investigaciones en la etapa de consolidación los elementos observables son acotados y precisos. Esta relación (etapa-objetivos) advierte la dificultad que se genera en la comprensión puntual tanto de la finalidad como del núcleo de acción de la innovación (Tejada, 1998), lo cual repercute en cada análisis en que la actividad de investigación vierte sus esfuerzos. En cuanto a las disciplinas a las que se dirigen las implementaciones, se observa el grueso de ellas asociado al aprendizaje de idiomas, sin embargo, las investigaciones más recientes empiezan a explorar su aplicación en asignaturas de carácter técnico y cada vez más bajo esquemas formales de educación.

Respecto a los enfoques teóricos de las investigaciones, se contemplan aspectos cognitivos, generacionales, motivacionales o de autorregulación. Modelos explicativos de otros enfoques como el de Koole o el de octalysis y con mayor acercamiento al microlearning, la espiral de aprendizaje de Baumgartner. El enfoque constructivista se presenta en algunas investigaciones, y de forma general, en todos los trabajos se abordan elementos teóricos que son propios del microlearning, aunque varían en la profundización que se hace de estos. Se trata de un alcance teórico diferenciado que permite colocar en debate la forma en cómo se han venido fundamentando las innovaciones relacionadas con el microlearning, sustento que tiende a adquirir relevancia 
en la determinación de procesos, procedimientos y estrategias que podrían facilitar la incorporación de la innovación en la enseñanza (Rivas, 2020).

La intención de este trabajo es comprender el desarrollo de innovaciones con relación a los enfoques teóricos que buscan ofrecer un sustento analítico al microlearning, considerando para ello las fases de un proceso innovador. Esta perspectiva de organización y análisis recupera elementos distintos y ofrece una visión alterna a las propuestas que valoran atributos como el tema, la procedencia de la investigación o elementos de diseño (De Gagne, Woodward, Park, Sun y Yamane, 2019; Jahnke, Lee, Pham, He y Austin, 2020). Las revisiones que parten de clasificaciones por temas de investigación suelen ser más comunes para identificar tendencias o necesidades de investigación; en el caso de este estudio, la falta de profundidad en este aspecto podría ser una limitante. Por otra parte, el énfasis en los elementos teóricos de las investigaciones, que representa la mirada de la investigación, suele dejarse de lado al presentar este tipo de trabajos, cuando en realidad constituye el fundamento de la dimensión sustantiva de la innovación (Tejada, 1998), y específicamente a aquellos aspectos sobre los cuales se define un determinado proceso de mejoramiento en la enseñanza.

\section{ConClusiones}

Con medios digitales diversos, variados enfoques en el aprendizaje y su incorporación en esquemas formales e informales de educación, la revisión expone la versatilidad del microlearning desde una perspectiva de innovación. La diversidad se observa en las propuestas analizadas, las cuales involucran, por mencionar algunas, plataformas educativas, desarrollos móviles específicos, el uso de redes sociales o su despliegue en conjunto con analíticas predictivas. La variedad en formatos comprende desde tweets e imágenes con contenidos concisos hasta ejercicios breves de evaluación, infográficos, videos y podcasts. Respecto a modalidades de aprendizaje, sus posibilidades son también variadas, se integra con ambientes blended, MOOCs, aula invertida, o con el aprendizaje a lo largo de la vida; enfoques como el constructivista y el aprendizaje situado también convergen en esta metodología, derivando en una matriz de posibilidades de innovación bastante amplia.

Por otra parte, el marco de análisis muestra que, según el momento en el que se sitúan las investigaciones en el proceso de innovación, la mayor parte de los esfuerzos se ubican en la etapa de gestación, de los que sobresalen los trabajos de aporte teórico, a partir de investigaciones documentales. Con relación al interior de las etapas de la innovación, la subclasificación que concentra más investigaciones es la de viabilidad material, comprendida en la etapa de ejecución. De estos estudios se destaca el uso escaso de la teoría, más bien se remiten a descripciones o atributos del microlearning. 
Bajo el recorte temporal que se presenta, se observa un incremento en las investigaciones sobre microlearning en los últimos años, también el enfoque de las investigaciones empieza a ser distinto, crecen las innovaciones en etapa de ejecución, mientras que en recortes temporales previos las investigaciones del tema se concentran con mayor contundencia en la etapa de gestación. Se hace notar cierta tendencia a establecer viabilidades para la incorporación de microlearning a los entornos virtuales de la educación superior, sobre todo en la producción científica del último año, en la cual se da especial énfasis a las posibilidades de la metodología con relación al contexto del COVID-19.

Puesto que la pandemia extendió el uso de entornos virtuales, ahora los resultados invitan a repensar con mayor detenimiento la instrucción a través de la virtualidad y a valorar alternativas con fundamento para la enseñanza-aprendizaje. La perspectiva de innovación adoptada en esta investigación aporta elementos teóricos que dan sustento al microlearning para el aprendizaje en la educación virtual. También sale a relucir la movilidad asociada a esta metodología, que destaca aún más que el uso de tecnología, por tanto, el desarrollo de contenidos tiende a ser adaptable a dispositivos móviles, flexibilizando el aprendizaje. Esta tendencia es favorable para los estudiantes de educación superior, visto que el acceso a tecnología móvil es más asequible en relación con otros dispositivos.

Las innovaciones analizadas describen un área de investigación cuyos aportes son aún limitados, con mayor contundencia en el ámbito de la educación superior. Por sus características, el microlearning ha tenido un mayor impacto y desarrollo en la educación no formal, asimismo las investigaciones dirigidas a ello han sido las de mayor interés. En el caso de la educación superior, el escaso fundamento empírico para estas innovaciones podría en cierto modo limitar su viabilidad al carecer de pautas y elementos, no solo técnicos sino sobre todo instruccionales, que permitan contextualizar su despliegue. Asimismo es de considerar que, como en toda innovación, habrá otras dimensiones de influencia en la consolidación y diseminación de esta, al menos en los escenarios y actores de la educación superior.

Finalmente, y a partir de estos hallazgos, se identifica la necesidad y la relevancia de indagar acerca de la disposición del estudiante hacia las interacciones con microlearning, asumida como innovación instruccional en los entornos virtuales de la educación superior. Esto se sustenta en que, de manera particular, el análisis y la perspectiva evolutiva de los avances en microlearning presentan las bases para fundamentar innovaciones instruccionales. Luego de lo experimentado al enfrentar la migración a un escenario de educación virtual, la reflexión gira en torno al aprendizaje de los estudiantes y a desarrollar su autonomía. No se trata solo de dar continuidad a la educación, sino de reconocer, concretar y dar fundamento a las posibilidades de innovación instruccional a través de la tecnología. La instrucción a través de entornos 
virtuales habrá de transitar a una didáctica que contemple este tipo de innovaciones, necesarias desde la perspectiva de los estudiantes, quizás más proclives a este tipo de aprendizajes por la interacción tecnológica creciente a la que están expuestos.

\section{REFERENCIAS}

Aguerrondo, I. (1992). La innovación educativa en América Latina: balance de cuatro décadas. Perspectivas: Revista Trimestral de Educación Comparada, (3), 381-394.

Allela, M. A., Ogange, B. O., Junaid, M. I., y Prince, B. C. (2019). Evaluating the effectiveness of a multimodal approach to the design and integration of microlearning resources in in-service teacher training. En Pan Commonweatlth Forum. Edinburgo, Escocia. Recuperado de: http://hdl.handle.net/11599/3272.

Almazova, N., Rogovaya, Y., y Gavrilova, A. (2018). Prospects of introduction of microlearning into the process of teaching postgraduate students a foreign language. En INTED2018 Proceedings: 12th International Technology, Education and Development Conference (pp. 3175-82). Doi: https://doi.org/10.21125/ inted.2018.0608.

Becker, S. A., Brown, M., Dahlstrom, E., Davis, A., DePaul, K., Diaz, V., y Pomerantz, J. (2018). NMC borizon report: 2018 higher education edition. Louisville, CO: Educause. Recuperado de: https://library.educause.edu/ /media/files/library/2018/8/2018hor izonreport.pdf.

Busse, J., y Schumann, M. (2019). How to create micro learning content in enterprises? Bridging theory and practice for deriving didactical requirements. En Proceedings of the 2019 SIGED International Conference on Information Systems Education and Research (pp. 51-57). Munich, Alemania: AIS eLibrary. Recuperado de: https://aisel.aisnet.org/cgi/viewcontent.cgi?article $=1005 \&$ context $=$ siged 2019 .

Coakley, D., Garvey, R., y O’Neill, Í. (2017). Microlearning adopting digital pedagogies to facilitate technology-enhanced teaching and learning for CPD. En Empowering 21st century learners through holistic and enterprising learning (pp. 237-242). Singapur: Springer. Doi: https://doi.org/10.1007/978-981-10-42416_24.

De Gagne, J. C., Woodward, A., Park, H. K., Sun, H., y Yamane, S. S. (2019). Microlearning in health pro- fessions education: a scoping review protocol. JBI Database of Systematic Reviews and Implementation Reports, 17(6), 1018-1025. Doi: https://doi.org/10.11124/ JBISRIR-2017-003884.

Educause (2020). 2020 Educause horizon report. Teaching and learning edition. Recuperado de: https://library. educause.edu/-/media/files/library/2020/3/2020_ horizon_report_pdf.pdf.

Fullan, M. (2013). Stratosphere. Integrating technology, pedagogy, and change knowledge. Toronto: Pearson.

Friedler, A. (2018). Teachers training micro-learning innovative model: Opportunities and challenges. En Proceedings of 2018 Learning with MOOCS (pp. 63-65). Doi: https://doi.org/10.1109/LWMOOCS.2018.8534647. Göschlberger, B. (2016). A platform for social microlearning. En K. Verbert, M. Sharples y M. Klobučar (eds.). European Conference on Technology Enhanced Learning EC-TEL (vol. 9891, pp. 513-516). Lyon, Francia: Springer. Doi: http://doi.org/10.1007/978-3-31945153-4_52.

Göschlberger, B., y Bruck, P. A. (2017). Gamification in mobile and workplace integrated microlearning. En Proceedings of the 19th International Conference on Information Integration and Web-based Applications \& Services (pp. 545-552). Doi: https://doi. org/10.1145/3151759.3151795.

Hug, T. (2012). Microlearning. En N. M. Seel (ed.). Encyclopedia of the sciences of learning. Boston, MA: Springer. Doi: https://doi.org/10.1007/978-1-4419-1428-6.

Jahnke, I., Lee, Y. M., Pham, M., He, H., y Austin, L. (2020). Unpacking the inherent design principles of mobile microlearning. Technology, Knowledge and Learning, 25(3), 585-619. Doi: https://doi.org/10.1007/ s10758-019-09413-w.

King, S. O. (2018). Microlearning: Using Twitter media to publish and facilitate engagement with innovative engineering programs. En Proceedings of the 2018 American Society for Engineering Education (ASEE) Southeastern Section Annual Conference. Recuperado de: 
https://sites.asee.org/se/wp-content/uploads / sites/56/2021/04/2018ASEESE29.pdf.

Lee, Y. J. (2008). A study of the influence of instructional innovation on learning satisfaction and study achievement. The Journal of Human Resource and Adult Learning, 4(2), 43-54. Recuperado de: http://www. hraljournal.com/Page/6\%20Yu-Je\%20Lee.pdf.

Meng, J., y Li, Z. (2016). Feasibility of applying mobile micro-learning to college English learning. En 2016 International Seminar on Education Innovation and Economic Management (SEIEM 2016). Atlantis Press. Doi: https://doi.org/10.2991/seiem-16.2016.123.

Meng, J., Wang, Z., y Li, Z. (2016). Application of cognitive load theory in mobile micro-learning. En 2016 International Conference on Management Science and Innovative Education. Atlantis Press. Doi: https:// doi.org/10.2991/msie-16.2016.110.

Nikou, S. A., y Economides, A. A. (2018). Mobile-based micro-learning and assessment: Impact on learning performance and motivation of high school students. Journal of Computer Assisted Learning, 34(3), 269-278. Doi: https://doi.org/10.1111/jcal.12240.

Pimienta, J., y De la Orden, A. (2017). Metodología de la investigación. México: Pearson.

Rivas, A. (2017). Cambio e innovación educativa: las cuestiones cruciales. Buenos Aires: Santillana.

Rivas, M. (2000). Innovación educativa: teoría, procesos y estrategias. Madrid: Síntesis.

Shail, M. S. (2019). Using micro-learning on mobile applications to increase knowledge retention and work performance: A review of literature. $\mathrm{Cu}$ reus, 11(8), e5307. Recuperado de: https://doi. org/10.7759/cureus.5307.
Shamir-Inbal, T., y Blau, I. (2020). Micro-learning in designing professional development for ICT teacher leaders: The role of self-regulation and perceived learning. Professional Development in Education, 1-17. Recuperado de: https://doi.org/10.1080/19415257.2020.1763434.

Skalka, J., y Drlik, M. (2020). Automated assessment and microlearning units as predictors of at-risk students and students outcomes in the introductory programming courses. Applied Sciences, 10(13), 4566. Doi: https://doi.org/10.3390/app10134566.

Tejada, J. (1998). Los agentes de la innovación en los centros educativos. Profesores, directivos y asesores. Málaga: Aljibe.

UNESCO [Organización de las Naciones Unidas para la Educación] (2020). Embracing a culture of lifelong learning. Contribution to the future of education initiative. Recuperado de: https://unesdoc.unesco.org/ark:/48223/ pf0000374112.

UNESCO-IESALC [Instituto Internacional de la UNESCO para la Educación Superior en América Latina y el Caribe] (2020). COVID-19 y educación superior: de los efectos inmediatos al día después. Caracas: UNESCO-IESALC. Recuperado de: http://www. iesalc.unesco.org/wp-content/uploads/2020/05/ COVID-19-ES-130520.pdf.

Zabalza, M. Á. (2004). Innovación en la enseñanza universitaria. Contextos Educativos. Revista de Educación, (6), 113-136.

Zhou, N., y Deng, Y. (2018). Research and practice on the flipped classroom teaching mode in "Microcomputer principle and interface technology" course based on the micro learning resources. International Journal of Information and Education Technology, 8(3). Doi: https:// doi.org/10.18178/ijiet.2018.8.3.1041.

Cómo citar este artículo:

Salas Díaz, F., González Bello, E. O., y Estévez Nénninger, E. H. (2021). Microlearning: innovaciones instruccionales en el escenario de la educación virtual. IE Revista de Investigación Educativa de la REDIECH, 12, e1262. doi: 10.33010/ ie_rie_rediech.v12i0.1262. 